\title{
ANALYSIS OF GENE COPY NUMBER VARIATIONS IN PATIENTS WITH CARDIAC SEPTAL DEFECTS USING MULTIPLEX LIGATION-DEPENDENT PROBE AMPLIFICATION
}

\author{
YASHVANTHI BORKAR ${ }^{1}$, KRISHNANANDA NAYAK ${ }^{2}$, RANJAN SHETTY $\mathrm{K}^{3}$, RAJASEKHAR MOKA ${ }^{1 *}$
}

${ }^{1}$ Department of Cell and Molecular Biology, Manipal School of Life Sciences, Manipal Academy of Higher Education, Manipal, Karnataka, India. ${ }^{2}$ Department of Cardiovascular Technology, Manipal College of Allied Health Sciences, Manipal Academy of Higher Education, Manipal, Karnataka, India. ${ }^{3}$ Department of Cardiology, Kasturba Hospital, Manipal Academy of Higher Education, Manipal, Karnataka, India. Email: rs.moka3@manipal.edu

Received: 26 October 2019, Revised and Accepted: 16 November 2019

\section{ABSTRACT}

Objective: Cardiac septal defects (CSDs), the most common human congenital heart malformations are complex and heterogeneous. Progress in molecular biology has helped to identify many genes responsible for cardiac morphogenesis. However, etiologic factors in familial as well as isolated syndromes are being identified; the root genetic cause still needs to be resolved and its mechanism is yet to be revealed. The objective of this study is to identify DNA copy number variations (CNVs) and their possible association with septal defects.

Methods: Multiplex ligation-dependent probe amplification (MLPA) was used to detect DNA copy number in non-syndromic CSDs using the P311-A1 Kit consisting of probes for the key genes, namely, NKX2-5 (NK2 transcription factor related, locus 5), GATA4 (GATA binding protein 4), TBX5 (T-box transcription factor), bone morphogenetic protein 4, and CRELD1 (cysteine rich with EGF-like domains 1).

Results: We studied 124 clinically diagnosed CSD subjects, of which 111 (89.5\%) had atrial septal defects and 13 (10.5\%) had ventricular septal defects. MLPA assay was carried out in all these patients after a thorough clinical and cytogenetic screening. CNVs were identified in 16 (12.9\%) cases, of which heterozygous deletions and heterozygous duplications were detected (8 patients each) with apparent phenotypes.

Conclusion: MLPA could be a useful assay for the detection of CNVs and to be adopted as the first line of screening in patients with congenital heart diseases.

Keywords: Copy number variations, Atrial septal defects, Ventricular septal defects, Multiplex ligation-dependent probe amplification, Heterozygous deletions, Heterozygous duplications.

(c) 2020 The Authors. Published by Innovare Academic Sciences Pvt Ltd. This is an open access article under the CC BY license (http://creativecommons. org/licenses/by/4. 0/) DOI: http://dx.doi.org/10.22159/ajpcr.2020.v13i1.36189

\section{INTRODUCTION}

Cardiac septal defects (CSDs) constitute $1 \%$ of all congenital heart diseases (CHDs) [1]. The prevalence of CHD worldwide (population based) is between 1 and 150/1000 live births, while in India, it is $8.07 / 1000$ live births [2]. However, septal defects have a reported frequency of 4.1/1000 live births. CHDs can either be isolated (nonsyndromic; septal defects) or in combination with other cardiac lesions (syndromic; Holt-Oram syndrome). The septal defects such as atrial septal defects (ASDs) and ventricular septal defects (VSDs) may be asymptomatic, with the appearance of murmur as the primary symptom. Many small septal defects close on their own as the heart grows; hence, percentage diagnosis that is typically made during childhood is low, except for a few which persist through adulthood. The diagnosis is usually based on echocardiography and transesophageal echocardiography. Congenital septal defects are the most common of all structural malformations of the human heart and are caused by both genetic and environmental factors [3]. Since the year 2000, several genetic epidemiological studies have confirmed its genetic predisposition [4]. Subsequent advancements in the molecular diagnostics helped in identifying genes responsible for cardiogenesis and causative of syndromic and non-syndromic forms of CHDs. Transcriptional factor genes are predominantly articulated in the heart tissue and are documented to have a physical interaction with other regulatory proteins for proper morphogenesis [5]. Despite recent molecular genetic studies, efforts to identify genotype-phenotype correlation in patients with septal defects suggest a more complex pattern of inheritance, where copy number variations (CNVs), as well as single nucleotide polymorphisms, contribute to the disease pathogenesis and tune the spectrum of septal malformations. CNVs contribute majorly toward the genetic diversity in context to evolution and disease susceptibility. Therefore, it is essential to detect the CNVs associated with CHDs. In this study, a multiplex ligation-dependent probe amplification (MLPA) assay was used to find out copy number changes in a cohort of 124 non-syndromic patients with CSDs. This MLPA assay is mainly used to utilize the many advantages provided when compared to other techniques. In fact, this assay allows a pool of samples to be handled simultaneously so that it could be used in high throughput analysis to find out copy number changes. MLPA could be a promising technique for proper diagnosis, prognosis, and management of the patients and affected families.

\section{METHODS}

In this study, the patients were referred from the outpatient cardiology department of Kasturba Hospital, Manipal. Subjects were ascertained through echocardiography to confirm the diagnosis and the size of the septal defects. The patient's details were noted in a case proforma which includes parental age at pregnancy, parental consanguinity, maternal gestational history, family history, history of any occupational/environmental exposures, socioeconomic status, and a three-generation pedigree. A written informed consent was obtained from the patient/guardian. Both the cardiologist and the genetic counselor analyzed the assessment of clinical phenotypes. In patients with CNVs, clinical and laboratory evaluations of the parents were included in the study. 
Peripheral blood samples (5 ml) were collected from each participant to conduct both cytogenetic and MLPA analysis. GTG-banded karyotype ( $\geq 450$ bands) was performed according to the modified method [6]. Lymphocytes were cultured and stimulated with known mitogen (Phytohemagglutinin) for $72 \mathrm{~h}$. Metaphase was arrested with colchicine and cells harvested using $0.075 \mathrm{M} \mathrm{KCl}$ and Carnoy's mixture (methanol/ acetic acid [3:1]). The slides were stained, and metaphases were captured, and analysis was done using a Zeiss microscope and IKAROS software.

Genomic DNA was extracted from whole blood of recruited patients $(\mathrm{n}=124)$ as well as controls $(\mathrm{n}=17 ; 8$ males and 9 females) using classic phenol-chloroform and QIAamp ${ }^{\circledR}$ genomic DNA kit (QIAGEN) according to the manufacturer's instructions. MLPA assay was performed as per the manufacturer SALSA MLPA kit P311-A1 (MRC-Holland, Amsterdam, Netherlands) protocol. Hybridization, ligation, and amplification were performed as described by Schouten et al. [7]. Two microlitre of the amplified product were diluted (1:30) in $58 \mu \mathrm{l}$ of distilled water, of which $2 \mu \mathrm{l}$ was plated into a well with $30 \mu \mathrm{l}$ of a mixture of 1 part of formamide Hi-Di (Applied Biosystem) and 0.015 parts GeneScan 500 LIZ size standard (Applied Biosystem). Capillary electrophoresis was performed on AB 3130 DNA analyzer (Applied Biosystems) as per the standardized laboratory protocol.

GeneMapper software v4.0 (Applied Biosystem) was used to perform fragment sizing with the internal 500 LIZ size standard, automated peak calling, and peak normalization. Thirty-five peak heights of 35 probes (linked to GATA4, NKX2-5, TBX5, BMP4, CRELD1, and DGCR8 genes) of each sample were exported for further data analysis. The peak heights were normalized by dividing each peak height by the sum of all 35 peak heights in each sample. Each normalized peak height was divided by the means of that peak height in the control probe. A spreadsheet was developed in Microsoft Excel to process the data for the control samples efficiently.

\section{RESULTS}

A total of 124 subjects (54 males and 70 females) with septal heart defects, aged between 3 (children) and 65 years (adult) registered at Cardiology OPD, Kasturba Hospital, Manipal. A case proforma was maintained to gather the clinical and family history which was used to analyze the correlation data. VSDs are the most common of all congenital cardiac anomalies, whereas in our study, ASDs were more common, due to a higher number of adult patients. The clinical phenotypes of these subjects are ASDs ( $\mathrm{n}=111)$ (Ostium secundum [OS]-ASD [n=102] and sinus venous [SV]-ASD [n=9]) and VSDs $(n=13)$ (perimembranous
[Pm]-VSD [n=7], muscular VSD [n=1], subpulmonic VSD [n=1], subaortic VSD [ $\mathrm{n}=1]$, and infravalvular gerbode VSD [ $\mathrm{n}=3]$ ). Familial recurrence was observed in $6 / 124$ patients, whereas consanguinity was seen in $18 / 124$ patients. Nearly $54.40 \%$ of the patients showed breathlessness and $58.4 \%$ had fatigue. A two-dimensional echocardiography was done in all patients; gradient and size of the septum were noted accordingly. Echocardiography revealed the thickness of either atrial or ventricular septum to be between $<10 \mathrm{~mm}$ and $50 \mathrm{~mm}(<10[\mathrm{n}=40$; $32.2 \%], 11$ and 20 [ $\mathrm{n}=49 ; 39.5 \%], 21$ and 30 [n=31; 25.0\%], 31 and $40[n=3 ; 2.41 \%]$, and 41 and $50[n=1 ; 0.80 \%])$. Family history and cardiac profiles such as electrocardiography, systolic functions, auricle dilation, right ventricular systolic pressure, and pulmonary arterial hypertension were also recorded. A cytogenetic study was carried out in every recruited patient as per the inclusion criteria to rule out gross chromosomal abnormalities and syndromes, and only isolated cases were included in the detection of CNVs.

MLPA analysis was done using the P311-A1 CHD SALSA MLPA Kit. Controls were run in the ratio 1:7 to the subject samples. A total of $16(12.9 \%)$ cases was found to have copy number changes in NKX2.5, GATA4, TBX5, BMP4, and DGCR8, as shown in Table 1. Heterozygous deletions were observed in eight patients and heterozygous duplications were observed in eight subjects with apparent phenotypes. The fragment separation was done using capillary electrophoresis, which employed an $\mathrm{ABI} 3130$ sequencer. The fragment analysis was performed using a trial version of Gene Marker software from SoftGenetics. The regions covered in the MLPA reactions using SALSA MLPA Kit P311-A1 are MSR1, CTSB, CRELD1, GATA4, TBX5, NKX2.5, GP1BB, BMP4, DGCR8, and CDC45 genes.

Sixteen CNVs (eight heterozygous deletions and eight heterozygous duplications) were identified in 124 patients studied with isolated CSD and the data are represented in Table 1. The following observations were derived from the data obtained. (1) The heterozygous duplication in exon 1 (5q35.1) of NKX2.5 was identified in an 18-year-old female with Pm-ASD phenotype; this patient had a family history of OS-ASD in both mother and elder sister. Interestingly, the same variation was detected with different phenotypes (OS-ASD) in two other patients, as shown in Table 1. (2) Heterozygous CNVs were found in five adult female patients, two cases of deletion in exon 1 (8p23.1) of GATA4 and three duplications in exon 6 of GATA4, of which four patients had OS-ASD while one had Pm-VSD. (3) Similarly, heterozygous CNVs in exon 9 (deletion) and exon 6 (duplication) in the TBX5 gene (12q2422) were detected in four patients with OS-ASD in three and PmVSD in one patient. (4) In the DGCR8 gene (22q11.21), we detected

Table 1: Clinical phenotypes and heterozygous variants identified in 16 subjects with heart septal defects

\begin{tabular}{|c|c|c|c|c|c|}
\hline S. No. & Age and gender & Clinical phenotype & Gene/chromosome position & GenBank exon & Results \\
\hline 1. & $18 / \mathrm{F}$ & Pm-VSD & NKX2.5 (5q35.1) & 1 & Heterozygous duplication \\
\hline 2. & $53 / F$ & OS-ASD & NKX2.5 (5q35.1) & 1 & Heterozygous duplication \\
\hline 3. & $66 / F$ & OS-ASD & NKX2.5 (5q35.1) & 1 & Heterozygous duplication \\
\hline 4. & $28 / F$ & OS-ASD & GATA4 (8p23.1) & 1 & Heterozygous deletion \\
\hline 5. & $50 / \mathrm{F}$ & OS-ASD & GATA4 (8p23.1) & 1 & Heterozygous deletion \\
\hline 6. & $23 / F$ & Pm-VSD & GATA4 (8p23.1) & 6 & Heterozygous duplication \\
\hline 7. & $41 / \mathrm{F}$ & OS-ASD & GATA4 (8p23.1) & 6 & Heterozygous duplication \\
\hline 9. & $11 / \mathrm{F}$ & OS-ASD & TBX5 (12q24.22) & 9 & Heterozygous deletion \\
\hline 10. & $36 / F$ & OS-ASD & TBX5 (12q24.22) & 9 & Heterozygous deletion \\
\hline 11. & $31 / \mathrm{M}$ & Pm-VSD & TBX5 (12q24.22) & 6 & Heterozygous duplication \\
\hline 12. & $40 / \mathrm{M}$ & OS-ASD & TBX5 (12q24.22) & 9 & Heterozygous duplication \\
\hline 13. & $9 / \mathrm{M}$ & OS-ASD & TBX5 (12q24.22) BMP4 (14q22.2) & 4,9 & Heterozygous deletion \\
\hline 14. & $4 / \mathrm{F}$ & Pm-VSD & TBX5 (12q24-22) BMP4 (14q22.2) & 4,9 & Heterozygous deletion \\
\hline 15. & $58 / \mathrm{M}$ & SV-ASD & DGCR8 (22q11.21) & 14 & Heterozygous deletion \\
\hline 16. & $24 / \mathrm{F}$ & SV-ASD & DGCR8 (22q11.21) & 14 & Heterozygous deletion \\
\hline
\end{tabular}

F: Female, M: Male, Pm-VSD: Perimembranous ventricular septal defects, OS-ASD: Ostium secundum atrial septal defect, SV-ASD: Sinus venosus atrial septal defect, NKX2-5: NK2 Transcription factor related, locus 5, GATA4: GATA binding protein 4, TBX5: T-box transcription factor, BMP4: Bone morphogenetic protein 4, DGCR8: DiGeorge syndrome critical region gene 8 
a heterozygous deletion in exon 14 in two patients with SV-ASD. (5) Two CNVs detected in this study were found to be associated with overlapping phenotypes, namely, the GATA4 (8p23.1) and the TBX5 (12q24-22) variations were found in patients with OS-ASD as well as Pm-VSD.

\section{DISCUSSION}

Septal defects can be caused by mutations in various transcriptional genes such as NKX2.5, GATA4, and TBX5. Synergic interactions of these genes are inevitable in cardiogenesis, particularly in the formation of chambers of the heart. Among mutations reported in these genes, CNVs including deletions and duplications are the most common in the general population with a frequency of $1-5 \%[8,9]$. Sometimes, benign CNVs may disrupt the reading frame of the gene and cause premature termination, causing splice site defects or even exon skipping. Genomic CNVs are playing a pivotal role in genetic architecture and well documented in many of the congenital abnormalities including heart defects and neurodevelopmental disorders such as developmental and intellectual disabilities $[10,11]$. Increasing knowledge of CNVs has moved focus toward a better understanding of CHDs [12]. Numerous techniques such as quantitative fluorescence polymerase chain reaction (PCR), MLPA, microarray, and next-generation sequencing have been described to quantify CNVs in human genetic disorders. However, MLPA assay has become one of the most widely used techniques to detect CNVs in several genetic diseases $[13,14]$. Several studies have shown the presence of recurrent CNVs detected in the patients with syndromic and non-syndromic CHD, including Sorensen's group [15] through MLPA screening.

In the present study, we detected copy number changes in 16 subjects (12.9\%) among 124 cases with septal defects who had a normal karyotype. Heterozygous deletions were identified in OS-ASD $(n=5)$, SV-ASD $(n=2)$, and Pm-VSD $(n=1)$. Heterozygous duplications were identified in Pm-VSD $(n=4)$ and OS-ASD $(n=5)$. However, none of these variations was inherited from the parents. However, duplications and deletions were observed in association with the same phenotypes. In various studies [16,17], similar phenotypes have been observed, where they speculated the variable expressions of the TBX5 gene. However, Liu et al. [18] revealed no evidence of GATA4 and NKX2.5 CNVs in the fetal CHD of the Chinese population except for the deletion in 22q11. Whereas, Guida et al. [19] revealed that individuals with isolated CHD screened for CNVs in their cohort shown had mutation-negative for GATA4. This may be due to the variation in geographical location and ethnicity. However, we found deletions and duplications in NKX2.5, GATA4, TBX5, and BMP4, as shown in the results.

We identified one deletion (148nt) and one duplication (238nt) in GATA4 at chromosomal position 8p23.1 in three OS-ASD and one Pm-VSD patient. As per the Database of Genomic Variants catalogue control population, no CNVs have been found to overlap with GATA4. Therefore, it is expected to conclude that septal defective phenotypes in these four individuals with CNVs at 8p23.1 may have resulted from the dosage sensitivity of GATA4 [20]. 8p23.1 duplication in GATA4 with variable phenotype, expressivity, and penetrance has been found to be associated with septal defects, conotruncal heart defects, developmental delay, and mild dysmorphism. Altered GATA4 dosage may vary due to variations in the TBX5 or other genes [21]. Interestingly, we detected one duplication each in 12q24.21 of TBX5 (346nt) in one OS-ASD and one Pm-VSD patient. NKX2.5 duplication has been observed at $5 \mathrm{q} 35.1 \mathrm{in}$ two OS-ASD patients (274 nt) and one Pm-VSD patient. In the case of Pm-VSD, NKX2.5 duplications were detected at two different regions (190 nt and $274 \mathrm{nt}$ ). We also detected a BMP4 deletion (391 nt) at 14q22.2 in two patients: One with OS-ASD and another with Pm-VSD; these two cases also showed a deletion in TBX5 (303 nt). In this study, we detected a 22q11.21 deletion (382 nt) in the region DGCR8 in two patients with SV-ASD. A similar CNV was reported earlier by Liu's group [18] in a patient with Tetralogy of Fallot.

\section{CONCLUSION}

Septal defects are a complex trait as multiple factors have been implicated in their pathogenesis, but the etiology has a strong genetic component, as shown by extensive epidemiological studies. The present study derives an inference that heterozygous deletions/duplications cause the incomplete formation of the cardiac septum and may lead to structural defects. This may be attributed to the misregulation of messenger RNA and protein synthesis during embryonic heart development. We, therefore, hypothesize that the loss/gain of transcriptional activity may cause structural and functional defects in the postnatal heart. Here, MLPA was used for the detection of microdeletions/duplications, due to its advantages over PCR and array comparative genomic hybridization, concerning practical simplicity and speed. MLPA is easy to implement in a standard diagnostic laboratory. In addition, any genomic region associated with CHDs can be targeted specifically using MLPA.

\section{ACKNOWLEDGMENT}

This work was ostensibly supported by the Indian Council for Medical Research (ICMR), New Delhi (F. No. 54/23/2012-HUM-BMS). We thank Director, Manipal School of Life Sciences, Manipal Academy of Higher Education, Manipal, for their support and infrastructure to carry out this work. We are also thankful to clinicians and colleagues for their cooperation and assistance. Finally, our appreciation is due to the patients and their families.

\section{AUTHORS' CONTRIBUTIONS}

Dr. Yashvanthi Borkar, Senior Research Fellow, preparation of the manuscript. Dr. Krishnananda Nayak, a technologist, provided the details of Echocardiography. Dr. Ranjan K Shetty, clinical cardiologist made the clinical diagnosis of every patient and reviewed the manuscript. Dr. Rajasekhar Moka supervised the manuscript preparation and review.

\section{CONFLICTS OF INTEREST}

The authors have no conflicts of interest to declare in relation to this article.

\section{REFERENCES}

1. Hoffman JI, Kaplan S. The incidence of congenital heart disease. J Am Coll Cardiol 2002;39:1890-900.

2. Saxena A, Mehta A, Sharma M, Salhan S, Kalaivani M, Ramakrishnan S, et al. Birth prevalence of congenital heart disease: A cross-sectional observational study from North India. Ann Pediatr Cardiol 2016;9:205-9.

3. Dietz H. Genetic epidemiology. In: Ferencz C, Rubin JD, Loffredo CA, Magee CA, editors. Epidemiology of Congenital Heart Disease: The Baltimore Washington Infant Study 1981-1989. Vol. 11. Mount Kisco, NY: Futura Publishing Company; 1994. p. 455-6.

4. Pierpont ME, Basson CT, Benson DW Jr., Gelb BD, Giglia TM, Goldmuntz E, et al. Genetic basis for congenital heart defects: Current knowledge: A scientific statement from the American Heart Association Congenital Cardiac Defects Committee, Council on Cardiovascular Disease in the Young: Endorsed by the American Academy of Pediatrics. Circulation 2007;115:3015-38

5. Granados-Riveron JT, Pope M, Bu'lock FA, Thornborough C, Eason J, Setchfield K, et al. Combined mutation screening of NKX2-5, GATA4, and TBX5 in congenital heart disease: Multiple heterozygosity and novel mutations. Congenit Heart Dis 2012;7:151-9.

6. Moorhead PS, Nowell PC, Mellman WJ, Battips DM, Hungerford DA. Chromosome preparations of leukocytes cultured from human peripheral blood. Exp Cell Res 1960;20:613-6.

7. Schouten JP, McElgunn CJ, Waaijer R, Zwijnenburg D, Diepvens F, Pals G. Relative quantification of 40 nucleic acid sequences by multiplex ligation-dependent probe amplification. Nucleic Acids Res 2002;30:e57.

8. Iafrate AJ, Feuk L, Rivera MN, Listewnik ML, Donahoe PK, Qi Y, et al. Detection of large-scale variation in the human genome. Nat Genet 2004;36:949-51.

9. Sebat J, Lakshmi B, Troge J, Alexander J, Young J, Lundin P, et al. Large-scale copy number polymorphism in the human genome. Science 2004;305:525-8 
10. Miller DT, Adam MP, Aradhya S, Biesecker LG, Brothman AR, Carter NP, et al. Consensus statement: Chromosomal microarray is a first-tier clinical diagnostic test for individuals with developmental disabilities or congenital anomalies. Am J Hum Genet 2010;86:749-64.

11. Cooper GM, Coe BP, Girirajan S, Rosenfeld JA, Vu TH, Baker C, et al. A copy number variation morbidity map of developmental delay. Nat Genet 2011;43:838-46.

12. Edwards JJ, Gelb BD. Genetics of congenital heart disease. Curr Opin Cardiol 2016;31:235-41.

13. Stuppia L, Antonucci I, Palka G, Gatta V. Use of the MLPA assay in the molecular diagnosis of gene copy number alterations in human genetic diseases. Int J Mol Sci 2012;13:3245-76.

14. John N, Rajasekhar M, Girisha KM, Sharma PS, Gopinath PM. Multiplex ligation-dependant probe amplification study of children with idiopathic mental retardation in South India. Indian J Hum Genet 2013; 19:165-70

15. Sørensen KM, El-Segaier M, Fernlund E, Errami A, Bouvagnet P, Nehme N, et al. Screening of congenital heart disease patients using multiplex ligation-dependent probe amplification: Early diagnosis of syndromic patients. Am J Med Genet A 2012;158A:720-5

16. Liao J, Kochilas L, Nowotschin S, Arnold JS, Aggarwal VS, Epstein JA, et al. Full spectrum of malformations in velo-cardio-facial syndrome/ DiGeorge syndrome mouse models by altering Tbx1 dosage. Hum Mol Genet 2004;13:1577-85

17. Borkar Y, Nayak K, Shetty RK, Moka R. A TBX5 nonsense mutation in siblings with divergent phenotypes associated with isolated septal defects. Asian J Pharm Clin Res 2017;10:126-30.

18. Liu Z, Wang J, Liu S, Deng Y, Liu H, Li N, et al. Copy number variation of GATA4 and NKX2-5 in Chinese fetuses with congenital heart disease. Pediatr Int 2015;57:234-8.

19. Guida V, Lepri F, Vijzelaar R, De Zorzi A, Versacci P, Digilio MC, et al. Multiplex ligation-dependent probe amplification analysis of GATA4 gene copy number variations in patients with isolated congenital heart disease. Dis Markers 2010;28:287-92.

20. Soemedi R, Wilson IJ, Bentham J, Darlay R, Töpf A, Zelenika D, et al. Contribution of global rare copy-number variants to the risk of sporadic congenital heart disease. Am J Hum Genet 2012;91:489-501.

21. Barber JC, Bunyan D, Curtis M, Robinson D, Morlot S, Dermitzel A, et al. 8p23.1 duplication syndrome differentiated from copy number variation of the defensin cluster at prenatal diagnosis in four new families. Mol Cytogenet 2010;3:3. 